L.E. Akpo ${ }^{1}$

M. Grouzis ${ }^{2}$

A. Gaston ${ }^{3}$

\title{
Pluviosité et productivité des herbages de l'aire pastorale de Wiidu Thiengoli au Ferlo (Nord Sénégal). Estimation des charges fréquentielles
}

\begin{abstract}
AKPO(L.E.), GROUZIS (M.), GASTON (A.). Pluviosité et productivité des herbages de l'aire pastorale de Wiidu Thiengoli au Ferlo (Nord Sénégal). Estimation des charges fréquentielles. Revue Élev. Méd. vét. Pays trop., 1993, 46 (4) : 675-681

Les caractéristiques pluviométriques et leurs influences sur la production des herbages ont été étudiées à partir de 15 années d'observation (1974-1988) autour du forage de Wiidu Thiengoli situé dans le Nord Sénégal. Les résultats font apparaître une très forte variabilité temporelle des précipitations et de la production. Un modèle simple de simulation de cette production en fonction de la pluviosité a été élaboré. Les charges fréquentielles établies à partir de cette formulation et de la série chronologique des pluies obtenues à Wiidu Thiengoli, comparées aux charges actuelles, font apparaître un déficit en disponible fourrager d'autant plus important que la période est sèche. Ce type de situation représente une menace pour les groupes pastoraux exploitant ce forage car la végétation naturelle constitue la quasi-totalité de l'alimentation des troupeaux dans ces systèmes de production.
\end{abstract}

Mots-clés : Parcours - capacité de charge - Pluie - Pâturage - Transhumance - Sahel - Sénégal.

\section{INTRODUCTION}

Dans les milieux sahéliens, où la quantité d'herbe produite pendant les deux à trois mois de la saison des pluies doit assurer l'alimentation du cheptel durant la longue période sèche, la notion de bilan fourrager (6), qui conditionne la capacité de charge, constitue un outil de choix pour améliorer l'exploitation des ressources. L'évaluation du disponible fourrager, réalisée à partir de la mesure de la production végétale herbacée, nécessite des observations étendues et répétitives compte tenu de la grande variabilité interannuelle observée (23).

Pour éviter de telles mesures relativement longues et onéreuses, des modèles de production de phytomasse en fonction de la pluie ont été proposés $(3,5,7,8)$. II apparaît que ces modèles doivent être circonscrits pour être utilisables en termes prévisionnels. Le but de ce travail est donc d'élaborer un modèle simple d'estimation de la production des herbages en fonction des précipitations pour l'aire de desserte du forage de Wiidu Thiengoli dans le nord du Sénégal.

1. Faculté des Sciences (Biologie végétale), Université Cheikh Anta Diop, B.P. 5005, Dakar, Sénégal.

2. ORSTOM, Laboratoire d'Ecologie végétale, B.P. 1386, Dakar, Sénégal.

3. CIRAD-EMVT, 10, rue Pierre-Curie, 94704 Maisons-Alfort Cedex, France.

Reçu le 9.2.1993, accepté le 22.3.1994.

\author{
MATÉRIEL ET MÉTHODE
}

\section{La zone d'étude}

Wiidu Thiengoli, situé à $15^{\circ} 20^{\prime}$ de latitude nord et à $16^{\circ}$ $21^{\prime}$ de longitude ouest, présente les principaux traits du climat sahélien sensu stricto à savoir : précipitations annuelles comprises entre 200 et $400 \mathrm{~mm}$, forte variabilité des précipitations ( $C V=30$ à $40 \mathrm{p}$. 100), humidité relative de l'air très faible, températures élevées, forte évaporation, précarité des réserves en eau du sol (19).

Les sols sont de type ferrugineux peu lessivés avec une tendance vers les lessivés, sur matériaux sableux d'origine éolienne faiblement argileux compacts (22). L'horizon $A$, pauvre en matières organiques, peut atteindre $40 \mathrm{~cm}$. Cet état facilite sa remobilisation par le vent et la formation d'ergs (24). Dans les zones interdunaires, les sols sont bruns subarides intergrades hydromorphes sur sables colluviaux. Pendant la saison des pluies, l'eau s'accumule dans les dépressions formant des mares temporaires.

La région de Wiidu Thiengoli est située dans le domaine phytogéographique des steppes à épineux où prédominent les thérophytes et les phanérophytes. La nette dominance de ces types biologiques traduit une bonne adaptation écologique aux sévères conditions écologiques d'aridité qui règnent dans le milieu (15)

Balanites aegyptiaca, Acacia seyal, A. senegal, Combretum glutinosum, Sclerocarya birrea sont les principales espèces ligneuses du groupement végétal sur pénéplaine haute (30). Ces populations de Sclerocarya birrea, arbre caractéristique des savanes autour de Wiidu, ainsi que celles de Combretum glutinosum et de Acacia senegal ont été particulièrement atteintes par la sécheresse persistante des deux dernières décennies $(25,29)$.

La végétation herbacée, essentiellement à base de graminées annuelles (Schoenefeldia gracilis, Cenchrus biflorus, Aristida mutabilis ) dépasse rarement $40 \mathrm{~cm}$ de hauteur. Les légumineuses sont représentées principalement par Zornia glochidiata, Alysicarpus ovalifolius et Indigofera senegalensis.

Deux types de parcours ont été identifiés (30) au niveau du groupement végétal sur pénéplaine haute : le parcours à Schoenefeldia gracilis et Aristida mutabilis 
(PS3) et le parcours à Diheteropogon hagerupii et Tephrosia purpurea (PS1).

L'aire de desserte du forage est de l'ordre de $700 \mathrm{~km}^{2}(15$ $\mathrm{km}$ de rayon). On a évalué à $20 \mathrm{p}$. 100 la surface occupée par l'ensemble des infrastructures et assimilé les 80 p. 100 restant au seul parcours PS3, car il occupe plus de 50 p. 100 de l'aire (30).

\section{Le système de production}

Dans une région pastorale, le forage est l'aménagement le plus important. Au Sénégal le développement de l'hydraulique pastorale a permis entre 1950 et 1980 la création de 68 points d'eau permanents. Le forage de Wiidu Thiengoli assure l'approvisionnement continu en eau de la population évaluée à 4100 habitants et répartie dans 20 localités (28). Les Peuhls y représentent plus de 80 p. 100.

Dans l'aire de desserte du forage, la pression qu'exercent les animaux sur le milieu dépend de leur effectif et de leur mode de conduite.

Les troupeaux y sont généralement mixtes : les Peuhls élèvent des bovins, des ovins et des caprins. II s'y ajoute des chevaux pour la selle et/ou le trait, des ânes pour les transports domestiques et quelquefois des dromadaires. Le dénombrement du cheptel, réalisé conjointement (sol et vol systématique de reconnaissance) par le Service de l'élevage et le Centre de suivi écologique (CSE) en 1991, donne la composition suivante : 9862 bovins, 10595 ovins, 4135 caprins, 150 asins, 260 équins et 147 camelins.

La création de ces forages (1954 pour Wiidu Thiengoli) a profondément perturbé les pratiques de ces sociétés pastorales qui ont observé un abandon progressif de la transhumance et un mouvement de sédentarisation (3). Les "rumano" (campements de saison des pluies) sont alors devenus des pôles de peuplement permanents. Entre 1950 et 1980, le cheptel a augmenté de 20 p. 100 et la proportion de transhumants est passée de 60 à 13 p. 100.

La gestion actuelle des parcours en saison pluvieuse repose sur des déplacements de faibles distances (moins de $2 \mathrm{~km}$ ) d'un "rumano" permanent vers une mare ; celleci assure un abreuvement quotidien du troupeau. La saison sèche voit l'exploitation des pâturages les plus élojgnés. Les campements de saison sèche ("sedano") sont éparpillés autour du forage à une distance allant de 2 à 5 $\mathrm{km}$ et peuvent être déplacés deux ou trois fois autour de celui-ci en fonction de la disponibilité des parcours.

La gestion actuelle du milieu consiste donc à essayer de maintenir le cheptel toute l'année sur les parcours compris à l'intérieur de l'aire de desserte du forage. Toutefois la mobilité existe toujours, mais au lieu d'être unidirectionnelle et périodique, elle est devenue anarchique. On passera plus à un micro-nomadisme de faible amplitude qu'à une véritable sédentarisation $(3,4)$.
Pendant les années à fort déficit pluviométrique ou lors de destruction accidentelle des pâturages par les feux de brousse, ou encore en cas de panne prolongée de la pompe du forage, on assiste à des transhumances exceptionnelles qui se manifestent par un exode quasi général vers le sud. Ainsi durant les 20 dernières années, les éleveurs de Wiidu Thiengoli n'ont quitté la zone que deux fois pour de grandes transhumances : au cours des sécheresses 1972-1973 et 1983-1984 (2).

\section{Les méthodes d'étude}

Les données relatives à la pluviosité ont été extraites des archives de la Météorologie nationale (1933 à 1982), des rapports annuels de la Mission forestière sénégalo-allemande (1982 à 1988) et de DEGOULET (10). Les données de phytomasse de 1974 à 1987 proviennent d'études agropastorales et de rapports du Service d'agrostologie du Laboratoire national d'élevage et de recherche vétérinaire (LNERV) de Dakar-Hann $(29,30)$. Ces données ont été complétées par des observations au cours de l'année 1988.

La production herbacée épigée est estimée, au maximum de végétation, par la méthode de la récolte intégrale, qui consiste à couper la strate herbacée au ras du sol dans des placeaux de 4 à $25 \mathrm{~m}^{2}$ (29), mais l'effectif de l'échantillonnage n'est pas toujours précisé dans les références, ce qui ne permet pas les calculs d'intervalle de confiance. Cette méthode est toutefois considérée comme très acceptable pour évaluer la productivité annuelle des communautés végétales à cycle court (27).

La production, exprimée en kilogrammes de matière sèche par hectare ( $\left.\mathrm{kg} \mathrm{MS} \mathrm{ha}^{-1}\right)$ est obtenue après détermination de la teneur en eau (séchage à $85^{\circ} \mathrm{C}$ à l'étuve jusqu'à poids constant d'un échantillon composite d'environ $1 \mathrm{~kg}$ ).

La conversion de l'effectif du cheptel en UBT (Unité bétail tropical, bovin adulte de $250 \mathrm{~kg}$ de poids vif), réalisée pour l'ensemble des espèces recensées donne une'charge animale de 11513 UBT.

La production utilisable, les capacités de charge et d'accueil sont calculées selon les normes classiques: coefficient d'utilisation de un tiers, consommation de 6,25 kg MS. j-1.UBT-1 (6).

\section{RÉSULTATS}

\section{Les caractéristiques pluviométriques}

\section{Analyse de la pluviosité de Wiidu Thiengoli et élaboration d'une série chronologique}

A Wiidu Thiengoli, la pluviosité annuelle moyenne (19741988) est de $264 \pm 51 \mathrm{~mm}\left(p_{0,05}\right)$. La variabilité est assez forte ; en effet, la pluviosité de l'année 1975 (475 mm) est 
environ quatre fois plus élevée que celle de 1983 (120 $\mathrm{mm}$ ). Le coefficient de variation (100 x écart-type de l'échantillon/moyenne) est de 34,8 p. 100, ce qui est tout à fait comparable à ce qui est donné par BARRAL et al. (3) pour la station voisine de Linguère (29 p. 100). II se situe bien dans la gamme des valeurs habituellement rapportées par LE HOUÉROU (19) pour le Sahel (37 p. 100 pour des précipitations annuelles allant de 200 à 299 $\mathrm{mm}$ ). On peut en outre noter que l'écart type par rapport à cette moyenne ( $\mathrm{s}=92 \mathrm{~mm}$ ) correspond au déficit moyen (36 p. 100) de la période 1970-1981, évalué par BARRAL et al. (3), pour un certain nombre de stations sahéliennes encadrant le Ferlo.

Le poste pluviométrique de Wiidu Thiengoli ne datant que de 1974 ne nous permet pas d'effectuer une étude fréquentielle des pluies. II nous faut établir une série chronologique plus longue en passant par une station voisine dans la même zone écologique. Pour cela, une régression a été calculée entre les pluviosités annuelles de Wiidu Thiengoli et celles de Linguère, habituellement utilisées pour caractériser la pluie du Ferlo $(3,29,30)$, Dagana plus au nord et Podor au nord-est.

La meilleure corrélation $\left(P_{w}=1,0 P_{D}+53,5\right)$ est obtenue avec la station de Dagana. Celle-ci explique en effet plus de 90 p. $100\left(\mathrm{r}^{2}\right)$ de la variabilité de la pluviosité de Wiidu Thiengoli. Podor n'en explique que 70,5 p. 100 alors que la corrélation n'est pas significative $\left(r^{2}=48,4\right.$ p. 100) avec les données de Linguère.

Le gain ( $n$ ') ou le nombre d'années d'extension de cette régression, calculé par la méthode proposée par DUBREUIL (14), est de 48 ans, c'est-à-dire que les pluviosités annuelles de Wiidu Thiengoli peuvent être estimées à partir de 1941.

\section{Analyse fréquentielle de la série chronologique.}

Les précipitations annuelles estimées de Wiidu Thiengoli ont été ajustées par la méthode du maximum de vraisemblance (logiciel PLUVIUM, ORSTOM) aux lois de Gauss et de Goodrich (fig.1). Le meilleur ajustement est obtenu avec la loi de Gauss. On en déduit les valeurs caractéristiques des pluies fréquentielles (tabl.I). La comparaison de ces pluies fréquentielles à la série chronologique (tabl. II) permet de noter que :

- les périodes humides $(P \geqslant 331 \mathrm{~mm})$ sont relativement rares (13 sur 48 ) soit environ 27,1 p. 100 ;

- les années exceptionnellement sèches $(1983,1984)$ sont également rares. Elles ont une récurrence de 50 ans ;

- les années présentant des précipitations annuelles de $264 \mathrm{~mm}$, qui correspondent à la moyenne observée pendant la période d'observation effective, sont assez fréquentes (tous les trois ans).

Cette étude montre en outre une énorme variabilité. Cette variabilité interannuelle des précipitations agit sur la structure des groupements végétaux herbacés, qui conditionne la production.
TABLEAU I Pluies fréquentielles ( $\mathrm{mm}$ ) d'après la loi de Laplace-Gauss à Wiidu Thiengoli (Nord-Sénégal)

\begin{tabular}{|c|c|c|c|c|}
\hline Périodes & Probabilités & Récurrence & Pluies $(\mathrm{mm})$ & $\pm \mathrm{IC}$ \\
\hline & 0,01 & 100 & 96,6 & 16,8 \\
& 0,02 & 50 & 120,2 & 17,0 \\
Sèche & 0,05 & 20 & 155,6 & 17,3 \\
& 0,10 & 10 & 187,0 & 17,6 \\
& 0,20 & 5 & 225,1 & 17,8 \\
& 0,35 & 3 & 264,6 & 18,0 \\
\hline Normale & 0,50 & 2 & 298,0 & 19,5 \\
\hline \multirow{5}{*}{ Humide } & 0,35 & 3 & 331,3 & 18,0 \\
& 0,20 & 5 & 370,4 & 17,8 \\
& 0,10 & 10 & 408,9 & 17,6 \\
& 0,05 & 20 & 440,4 & 17,3 \\
& 0,02 & 50 & 475,8 & 17,0 \\
& 0,01 & 100 & 499,4 & 16,8 \\
\hline
\end{tabular}

TABLEAU II Pluviosité de Wiidu Thiengoli (1941-1988)

\begin{tabular}{|c|c|c|c|c|c|}
\hline Années & $\begin{array}{c}\text { Pluies } \\
(\mathrm{mm})\end{array}$ & Années & $\begin{array}{c}\text { Pluies } \\
(\mathrm{mm})\end{array}$ & Années & $\begin{array}{c}\text { Pluies } \\
(\mathrm{mm})\end{array}$ \\
\hline 1941 & 189,2 & 1957 & 438,0 & 1973 & 274,0 \\
1942 & 280,4 & 1958 & 371,0 & 1974 & 239,0 \\
1943 & 316,6 & 1959 & 206,0 & 1975 & 315,0 \\
1944 & 240,3 & 1960 & 381,0 & 1976 & 342,0 \\
1945 & 240,5 & 1961 & 365,0 & 1977 & 233,0 \\
1946 & 316,2 & 1962 & 273,0 & 1978 & 287,0 \\
1947 & 225,0 & 1963 & 380,0 & 1979 & 202,0 \\
1948 & 296,0 & 1964 & 329,0 & 1980 & 197,0 \\
1949 & 349,5 & 1965 & 341,0 & 1981 & 193,0 \\
1950 & 294,0 & 1966 & 450,0 & 1982 & 184,0 \\
1951 & 410,0 & 1967 & 390,0 & 1983 & 120,0 \\
1952 & 415,0 & 1968 & 213,0 & 1984 & 131,0 \\
1953 & 384,0 & 1969 & 422,0 & 1985 & 302,0 \\
1954 & 311,0 & 1970 & 268,0 & 1986 & 349,0 \\
1955 & 282,0 & 1971 & 229,0 & 1987 & 281,0 \\
1956 & 327,0 & 1972 & 134,0 & 1988 & 335,0 \\
\hline
\end{tabular}

\section{L'analyse de la production des herbages}

\section{La phytomasse}

La figure 2 représente les variations interannuelles de la phytomasse herbacée aérienne au cours de la période d'observation dans l'aire de desserte du forage de Wiidu Thiengoli. La valeur moyenne (1974-1988) s'élève à 750

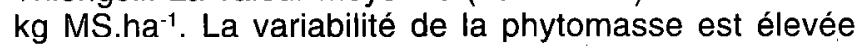
puisqu'elle varie de $1300 \mathrm{~kg} \mathrm{MS} \mathrm{ha}^{-1}$ (1975) à $112 \mathrm{~kg}$ MS.ha ${ }^{-1}$ en 1984 (soit un rapport de 1 à 13) alors que celle de la pluviosité se situe dans le rapport 4 . Le rapport de la variabilité de la production à celle de la pluviosité qui se situe donc à plus de 3 est donc deux fois plus 


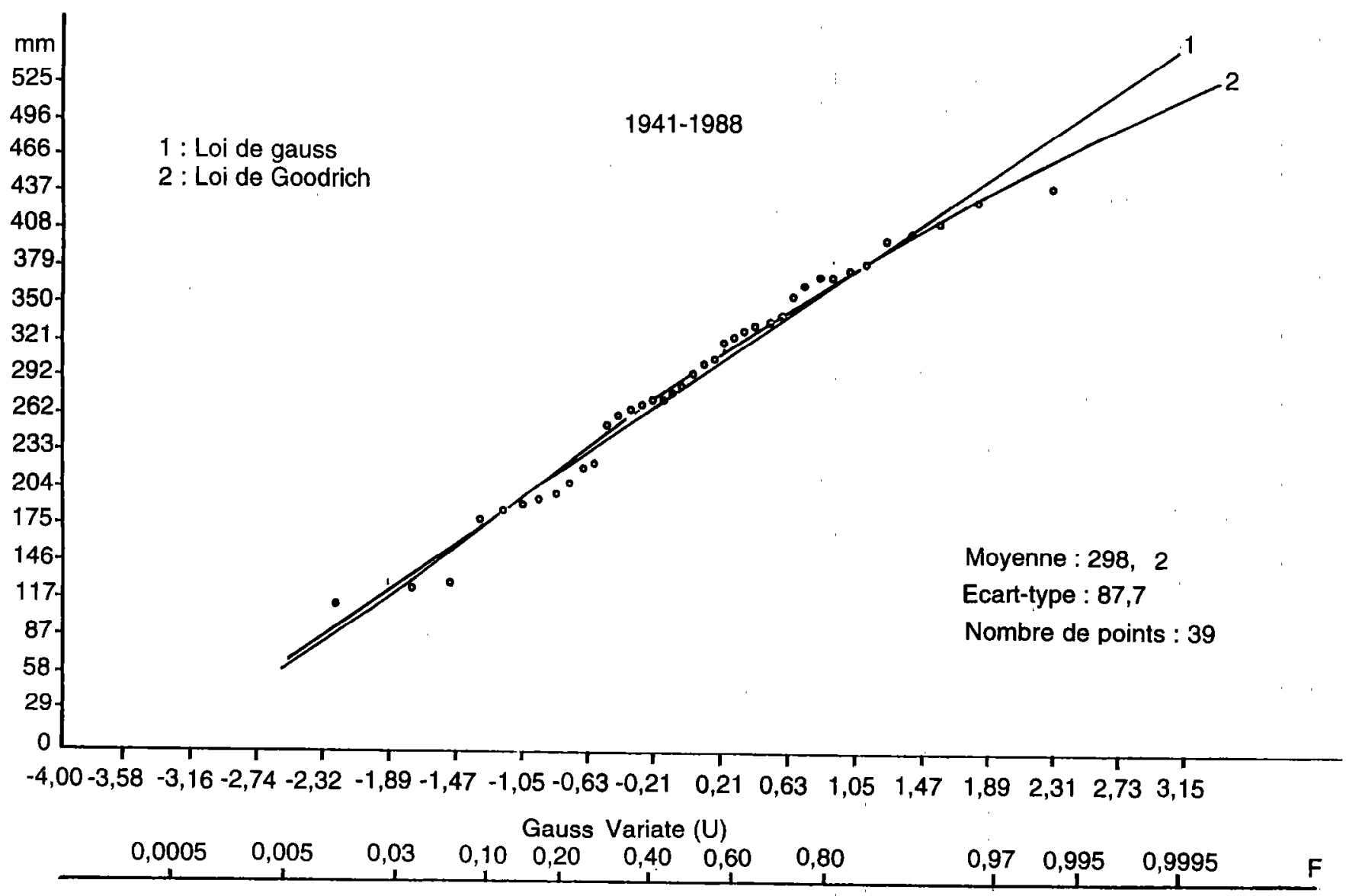

Figure 1. Ajustement des précipitations annuelles estimées de Wiidu Thiengoli (1941 à 1988) aux lois de Gauss et de Godrich.

important que celui indiqué par LE HOUÉROU (19), LE HOUÉROU et al. (21).

La relation entre la production de la strate herbacée des parcours $\mathrm{P}\left(\mathrm{kg} \mathrm{MS}^{\mathrm{h}} \mathrm{ha}^{-1}\right.$ ) et les précipitations annuelles $\mathrm{P}$ $(\mathrm{mm})$ a été établie (fig. 3). L'équation de la droite est :

$$
P\left(\mathrm{~kg} \mathrm{MS.ha}^{-1}\right)=3,0 \mathrm{P}(\mathrm{mm})-41,8\left(\mathrm{r}^{2}=0,64\right)
$$

Les précipitations annuelles dans la région de Wiidu Thiengoli sont assez déterminantes, puisqu'elles expliquent plus de 60 p. $100\left(r^{2}\right)$ de la variance de la phytomasse. Comparé aux valeurs rapportées par divers auteurs $(3: 33,1$ p. 100 pour le Ferlo; $12: 25$ p. 100 pour certaines stations du Ferlo ; $17: 30$ à 37 p. 100 pour différents écosystèmes pastoraux arides et semi arides), le coefficient de détermination obtenu autour du forage de Wiidu Thiengoli est nettement plus élevé. Cela s'explique par le fait que la formulation s'applique à une aire restreinte, donc à une zone plus homogène sur le plan structural. L'intégration du nombre de jours de pluie dans une régression multiple améliore de $5 \mathrm{p}$. 100 l'estimation de la production de phytomasse.
L'expression de la phytomasse en fonction de la pluie permet de comparer le résultat obtenu avec d'autres travaux (tabl. III).

Ces données montrent qu'en zone sahélienne, les valeurs estimées de la production globale de phytomasse herbacée par millimètre de pluie incidente sont assez voisines : 2,5 à $4 \mathrm{~kg} \mathrm{MS}$.ha-1. $\mathrm{mm}^{-1}$. Cela témoigne du fonctionnement comparable des végétations sahélo-soudaniennes. Le résultat obtenu à Wiidu Thiengoli correspond pratiquement à la moyenne des différents résultats. La variabilité vient essentiellement de l'échelle d'investigation, parcellaire $(5,7,15)$ dans certains cas, à régionale dans d'autres $(9,20)$.

\section{Phytomasse herbacée et charges fréquentielles}

La capacité de charge est calculée pour quelques fréquences remarquables (tabl. IV) et pour une utilisation essentiellement de saison sèche de huit mois (novembre à juin) à partir de la production obtenue par le modèle :

$$
P\left(\mathrm{~kg} \mathrm{MS} \cdot \mathrm{ha}^{-1}\right)=3,0 \mathrm{P}(\mathrm{mm})-41,8
$$




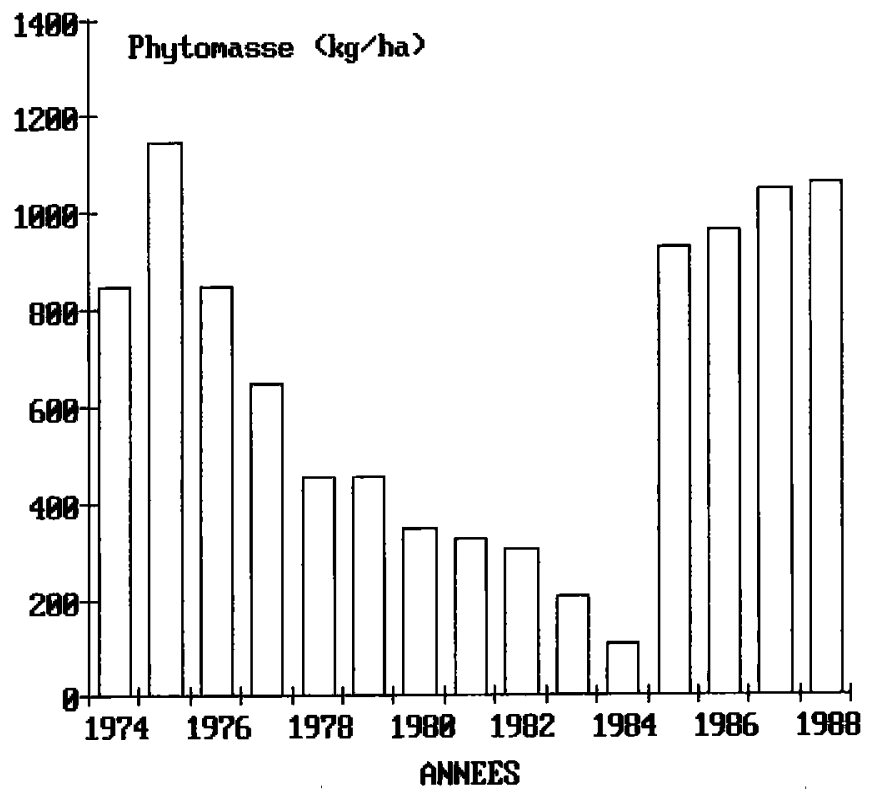

Figure 2. Variations interannuelles de la phytomasse herbacée épigée dans l'aire pastorale de Wiidu Thiengoli de 1974 à 1988.

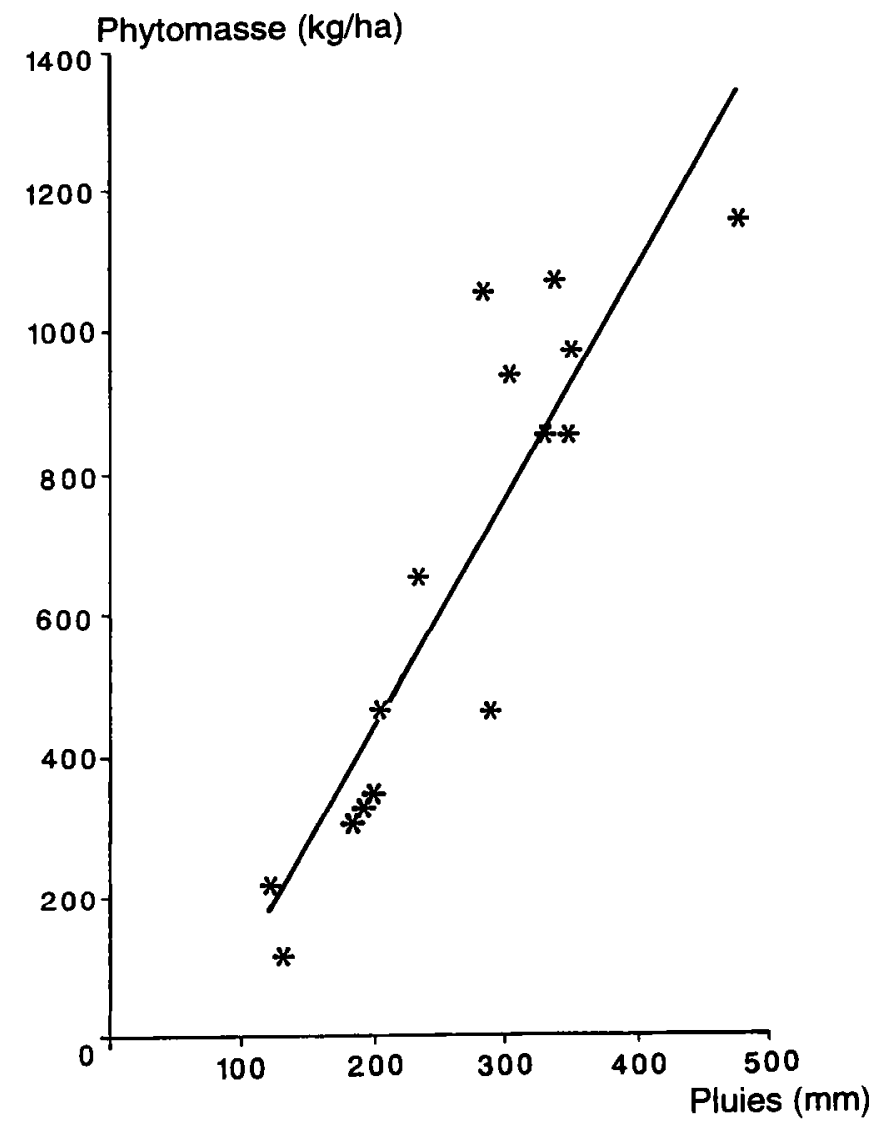

Figure 3. Relations entre la phytomasse herbacée épigée $P\left(\mathrm{~kg} M S . h a^{-1}\right)$ et la pluie incidente ( $\mathrm{mm}$ ).

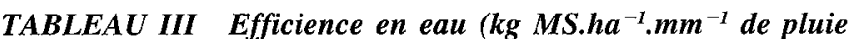
incidente) en zone sahélo-soudanienne.

\begin{tabular}{|l|c|}
\hline \multicolumn{1}{|c|}{ Auteurs } & $\begin{array}{c}\text { Production de matière sèche } \\
\text { (kg.ha }{ }^{-1} \cdot \mathrm{mm}^{-1} \text { pluie incidente) }\end{array}$ \\
\hline BARRAL et al. (3) & 3,77 \\
BILLE (5) & 2,32 \\
CORNET (17) & 3,30 \\
DE VRIES et DJITEYE (19) & 4,18 \\
DIARRA, BREMAN (11) & 2,40 \\
DIEYE (12) & 3,20 \\
GROUZIS (15) & 2,90 \\
LAMPREY, YUSSUF (17) & 2,22 \\
LE HOUEROU (18) & 3,70 \\
LE HOUEROU (20) & 2,70 \\
\hline Notre résultat & 3,07 \\
\hline
\end{tabular}

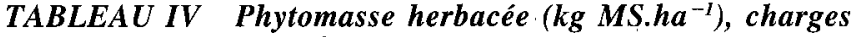
fréquentielles (ha.UBT ${ }^{-1}$ ) et capacité d'accueil (UBT) dans l'aire pastorale de Wiidu Thiengoli.

\begin{tabular}{|c|c|c|c|c|c|}
\hline \multirow{2}{*}{$\begin{array}{l}\text { Récur- } \\
\text { rence } \\
\text { (ans) }\end{array}$} & \multirow{2}{*}{$\begin{array}{l}\text { Pluies } \\
(\mathrm{mm})\end{array}$} & \multicolumn{2}{|c|}{ Production (kg MS.ha $\left.{ }^{-1}\right)$} & \multirow{2}{*}{$\begin{array}{l}\text { Charge } \\
\text { ha/UBT }\end{array}$} & \multirow{2}{*}{$\begin{array}{c}\text { Capacité } \\
\text { d'accueil } \\
\text { (UBT) }\end{array}$} \\
\hline & & totale & utilisable & & \\
\hline 100 & 96,6 & 247,0 & 84,2 & 18,2 & 3105 \\
\hline 50 & 120,2 & 317,6 & 105,9 & 14,2 & 3980 \\
\hline 20 & 155,6 & 423,5 & 141,2 & 10,6 & 5332 \\
\hline 10 & 187,0 & 517,3 & 172,4 & 8,7 & 6497 \\
\hline 5 & 225,1 & 631,3 & 210,4 & 7,1 & 7961 \\
\hline 3 & 264,6 & 749,4 & 249,8 & 6,0 & 9420 \\
\hline 2 & 298,0 & 849,2 & 283,1 & 5,3 & 10664 \\
\hline 3 & 343,3 & 984,7 & 328,2 & 4,6 & 12287 \\
\hline 5 & 370,8 & 1066,9 & 355,6 & 4,2 & 13457 \\
\hline 10 & 408,9 & 1180,8 & 393,6 & 3,8 & 14874 \\
\hline 20 & 440,4 & 1275,0 & 425,0 & 3,5 & 16149 \\
\hline 50 & 475,8 & 1380,8 & 460,3 & 3,3 & 17127 \\
\hline 100 & 565,5 & 1649,1 & 549,7 & 2,8 & 20186 \\
\hline
\end{tabular}

Les données du tableau IV font apparaître une capacité de charge de 5,3 ha.UBT ${ }^{-1}$ une année sur deux. Elle se situe bien dans la gamme des valeurs (5 à 6,5 ha.UBT-1) rapportées par VALENZA et DIALLO (30); sachant que les précipitations de 1971, année de référence, étaient inférieures de 12 p. 100 à la normale.

En utilisant la pluviosité estimée de 1971, la capacité de charge est identique. MIEHE-KLUG (25) trouve autour du même forage une capacité de charge de 5,6 ha.UBT ${ }^{-1}$. Ces comparaisons montrent que le modèle proposé est un bon estimateur de la production de phytomasse et de la capacité de charge, dans la zone considérée.

En comparant l'effectif actuel de la zone à la capacité d'accueil, on constate, une année sur deux, que la production herbacée autour du forage de Wiidu Thiengoli ne 


\section{L.E. Akpo M. Grouzis A. Gaston}

permet pas d'assurer l'entretien du cheptel présent évalué à 11500 UBT (1). Un délestage d'environ 10 p. 100 de l'effectif total serait théoriquement nécessaire pour assurer la couverture des besoins alimentaires des animaux.

Pour les années exceptionnellement sèches telles que 1983 et 1984, le délestage devrait être de l'ordre de $60 \mathrm{p}$. 100. Ce serait sur le niveau de production des années défavorables qu'il faudrait baser les plans d'aménagement d'un secteur d'élevage si l'on veut conserver le pâturage pour une exploitation durable.

Dans le cas de la centenale favorable (année particulièrement humide), même en tenant compte du supplément de 20 p. 100 apporté par la strate ligneuse (5) à la phytomasse herbacée, la production totale ne permettrait plus, dans une vingtaine d'années, d'assurer la couverture des besoins des animaux, dans l'hypothèse d'un taux d'accroissement de 2,5 p. 100 l'an (1), conduisant à un effectif de 28000 UBT. De plus, la fertilité des sols peut devenir limitante (9) dans des conditions favorables (PS $\geq 500 \mathrm{~mm}$ )

Ce modèle simple de production permet de déterminer la charge en bétail à partir de la pluie annuelle en passant par la production herbacée, et de décider, à la fin de la saison des pluies, de mesures de délestage de la zone en recourant à une transhumance élargie $(15,26)$, d'organisation de vente de bétail ou de complémentation pour ajuster la charge aux ressources de l'année.

\section{CONCLUSION}

\section{Déséquilibre, dégradation de l'espace}

A Wiidu Thiengoli, la capacité d'accueil en année normale (récurrence 2) est de 10700 UBT. L'excédent, par rapport à l'effectif actuel est de 800 UBT, soit 10 p. 100. Ce léger déséquilibre peut être aggravé par des apports saisonniers de bétail, notamment venant de Mauritanie ou suite à une panne d'un des forages voisins (Tatki, Tessékré, Belel Boguel), ainsi que par la destruction des pâturages par les feux de brousse. De plus, l'eau du forage de Wiidu Thiengoli a la particularité d'être chargée avec des résidus secs de l'ordre de $250 \mathrm{mg} / \mathrm{l}$; l'eau est donc de très bonne qualité, ce qui explique l'affluence des troupeaux, surtout en fin de saison sèche (2).

Pour mieux établir le bilan autour du forage de Wiidu Thiengoli, il serait donc utile de chiffrer l'importance de ces variations saisonnières et/ou interannuelles du bétail.

Ce relatif équilibre est vite rompu en année sèche. En effet, il apparaît, tous les trois ans, un excédent de charge de l'ordre de 20 p. 100 (2 100 UBT) par rapport à l'effectif du cheptel actuel. En années exceptionnellement sèches (1983-1984) l'excédent de charge est trois fois plus élevé. Il est vrai que pendant ces années fortement déficitaires, les éleveurs transhument. Cependant, les possibilités de déplacement vers les autres zones pastorales se raréfient ; des cultures irriguées en riz s'étendant sur de grandes superficies, gênent considérablement l'accès au fleuve Sénégal (26). A l'ouest, la voie vers le lac de Guiers est aussi barrée par des périmètres irrigués, tandis qu'au sud il y a fréquemment des conflits avec les agriculteurs. Au cours des 40 dernières années, les zones d'accueil ont donc considérablement diminué (2).

Notons aussi que le modèle est établi sur la base de la production moyenne d'un seul parcours (PS3) alors qu'il aurait fallu tenir compte du deuxième parcours (PS1).

Par ailleurs, bien que DIOP (13) considère qu'il existe des zones plus ou moins intensément exploitées, le modèle s'appuie sur une utilisation homogène de cet espace, puisque le micro nomadisme défini par BARRAL et al. (3) apparaît comme une stratégie pastorale parfaitement cohérente qui permet de tirer intégralement parti des pâturages les plus lointains à l'intérieur des aires d'influence des forages.

Même s'il existe un relatif équilibre, qu'il faudra d'ailleurs préciser par des analyses plus approfondies des effectifs du cheptel et de leurs variations, le déséquilibre est latent en raison notamment de :

- l'aridification du système lié à la sécheresse permanente depuis une vingtaine d'années (16);

- la dégradation des stratégies pastorales $(3,4)$;

- la réduction des zones d'accueil.

Dans ces conditions, il serait nécessaire, pour permettre le maintien des capacités de production de l'ensemble des éléments du système, c'est-à-dire pâturages et cheptel, de tenir compte dans les plans d'aménagement de ces aires des productions des fréquences pluviométriques défavorables et d'évaluer annuellement la production fourragère en fin de saison des pluies, à l'aide du modèle.

\section{REMERCIEMENTS}

Nous exprimons notre reconnaissance au personnel du service d'Agrostologie du Laboratoire national d'élevage et de recherches vétérinaires de Dakar-Hann pour son accueil au cours de l'année 1988. Nous remerçions également Messieurs J. ALBERGEL et H. DACOSTA du Laboratoire d'hydrologie du Centre ORSTOM de Dakar pour l'aide apportée au traitement des données pluviométriques. 


\section{BIBLIOGRAPHIE}

1. AKPO (L.E.). Dynamique des systèmes écologiques sahéliens. Structure spécifique, productivité et qualité des herbages : le forage de Wiidu Thiengoli (Nord Sénégal). DEA, Université de Dakar, 1990. 55 p.

2. AMADOU (L.). Concept de gestion des parcours des peulhs dans le Nord Sénégal. Rapport de mission d'étude. Kelkheim, GTZ, 1992. 61 p. + ann.

3. BARRAL (H.), BENEFICE (E.), BOUDET (G.), DENIS (J.P.), DE WISPELAERE (G.), DIAITE (I.), DIAW (O.T.), DIEYE (Kh.), DOUTRE (M.P.), MEYER (J.F.), NOEL (J.), PARENT (G.), PIOT (J.), PLANCHENAULT (D.), SANTOIR (C.), VALENTIN (C.), VALENZA (J.), VASSILIADES (G.). Systèmes de production d'élevage au Sénégal dans la région du Ferlo : synthèse de fin d'étude d'une recherche d'équipes pluridisciplinaires. Paris, GERDAT, ORSTOM, 1983. $112 \mathrm{p}$

4. BENOIT (M.). La lisière du Kooya : un espace pastoral et paysages dans le nord du Sénégal (Ferlo). Espace géogr., 1988, 2, : 95-108.

5. BILLE (J.C.). Etude de la production primaire nette d'un écosystème sahélien. PARIS, ORSTOM, 1977. 82 p. (Travaux et Documents, 65)

6. BOUDET (G.). Manuel sur les pâturages tropicaux et les cultures fourragères, 4e éd. Paris, La Documentation française, 1991. 266 p. (Manuels et Précis d'élevage $\mathrm{N}^{\circ} 4$ )

7. CORNET (A.). Le bilan hydrique et son rôle dans la production de la strate herbacée de quelques phytocénoses sahéliennes au Sénégal. Thèse Dr. Ing. Montpellier, U.S.T.L., 1981. 353 p.

8. CORNET (A.), RAMBAL (S.). Simulation de la production de l'eall et de la production végétale d'une phytocénose sahélienne du Sénégal. Oecol. Plant., 1981, 3 (17) : 381-397.

9. DE VRIES (F.W.T.), DJITEYE (M.A.), Eds. La productivité des pâturages sahéliens. Une étude des sols, végétations et de l'exploitation de cette ressource naturelle. Wageningen, $\mathrm{CABO}, 1982.525 \mathrm{p}$.

10. DEGOULET (A.). Etude des pluies journalières de fréquence rare au Mali, en Mauritanie et au Sénégal. Ouagadougou, CIEH, 1984. 129 p. (Sér. hydrologie)

11. DIARRA (L.), BREMAN (H.). Influence de la pluviosité sur la production des pâturages. $I n$ : Actes du Colloque de Bamako (Mali) "Inventaire et cartographie des pâturages tropicaux africains", 3-8 mars 1975. Addis Abeba, CIPEA. p. 171-174.

12. DIEYE (Kh.). Evaluation des ressources fourragères naturelles par le bilan hydrique. Cas du Ferlo sénégalais. In : Méthodes d'inventaire et de surveillance continue des écosystèmes pastoraux sahéliens. Application au développement. Actes du colloque tenu à Dakar, 16-18 novembre 1983. Ch. L. VANPRAET Ed. Dakar, 1983. p. 43-86.

13. DIOP (A.T.). La gestion des parcours de l'aire d'influence de Tatki relations entre données de végétation, taux d'exploitation et transhumance. Revue Élev. Méd. vét. Pays trop., 1992, 45 (1) : 81-90

14. DUBREUIL (P.). Initiation à l'analyse hydrologique. Paris. Masson, ORSTOM, 1974. $216 \mathrm{p}$.

AKPO(L.E.), GROUZIS (M.), GASTON (A.). Rainfall and grazing production of pastoral area of Wiidu Thiengoli (Ferlo, North Senegal). Evaluation of frequency rate of stocking. Revue Élev. Méd. vét. Pay's trop., 1993, 46 (4) : 675-681

The authors have studied the rainfall characteristics and the indice of grassland production from the date of a 15 year observation period (1974-1988) around the bore hole of Wiidu Thiengoli. The results show a very large time variability in precipitation and production. $A$ simple model of simulation of the production according to the rainfall level has been developped. The frequency rate of stocking established in relation to this formulation together with the chronological series of the rainfall at Wiidu Thiengoli give evidence of a forage deficiency which depends on the dryness of the season. This inadequacy represents a threat for the nomadic (pastoral) groups using the bore hole, as the natural vegetation is almost the only feding resource of the herds in this particular production system.

Key-words : Rangelands - Stocking rate - Rain - Grazing - Transhumance - Sahel - Senegal.
15. GROUZIS (M.). Structure, productivité et dynamique des systèmes écologiques sahéliens : la mare d'Oursi au Burkina Faso. Paris, ORSTOM, 1988. 366 p. (Coll. Etudes et Thèses)

16. GROUZIS (M.), ALBERGEL (J.), CARBONNEL (J.P.). Péjoration climatique au Burkina Faso : effets sur les ressources en eau et les productions végétales, In :"Les hommes face aux sécheresses, Nordeste brésilien, Sahel africain" HEAL EST-Samuel Tastet Ed., Paris, 1986. p. 165-178..

17. LAMPREY (H.F.), YUSSUF (H). Pastoralism and desert encroachment in Northern Kenya. Ambio, 1981. X : 131-134.

18. LE HOUÉROU (H.N.). Rain use efficiency : a unyfying concept in arid-land ecology. J. arid Env., 1984, $7: 1-35$.

19. LE HUUÉROU (H.N.). The grazing land ecosystems of the African Sahel. Paris, Springer Verlag, 1989.282 p. (Ecological Studies 75)

20. LE HOUÉROU (H.N.), HOSTE (C.H.). Rangeland production and animal rainfall relation in the Mediterranean and in the African sahelo-soudanian zone. J. Range Mgmt, 1977, 30 (3) : 181-189.

21. LE HOUÉROU (H.N.), BINGHAM (R.L.), SKERBEK (W.). Relationship between the variability of primary production and of annual precipitation in world arid land. J. arid Env. , 1988, $15: 1-18$.

22. LEPRUN (J.C.). Nouvelles observations sur les formations sablenses fixées du Ferlo occidental (Sénégal). Ass. sénégalaise Etud. Quaternaire Ouest afr., Bull. liaison, 1971, 31:69-78.

23. LEVANG (P.), GROUZIS (M.) Méthodes d'étude de la biomasse herbacée des formations sahéliennes: applications à la mare d'Oursi, HauteVolta. Acta oecologica, Oecol. Plant. 1980, 1 (3) : 231-244.

24. MAIGNEN (R.). Nutice explicative de la carte des sols du Sénégal. Paris, ORSTOM, 1965. ( 1 carte à $1 / 1000000$ ).

25. MIEHE-KLUG (S.). Inventaire et suivi (monitoring) de la végétation dans les parcelles à Wiidu Thiengoli (Ferlo, Sënégal). Hamburg, GTZ, 1990.

26. Profil de l'environnement de la vallée du fleuve Sénégal. DGCD/MAE, Euroconsult/RIN, $1990.68 \mathrm{p}$.

27. SING (J.S.), LAUENROTH (W.K.), STEINHORST (R.K.). Rewiew and assessement of various technics for estimating net aerial primary production in grasslands from harvest data. Bot. Rev.,1975, 41 (2) : 181-232.

28. TLUCZYKONT (S.), RICHTER (M.), KASBERGER-SANFTL (G.). Le pâturage contrôlé : un systc̀mc d'cxploitation sylvo-pastorale comme modèle pour la sauvegarde des ressources naturelles. Saint-Louis (Sénégal), Mission forestière allemande, $1991.100 \mathrm{p}$. + annexes.

29. VALENZA (J.). Surveillance continue des pâturages naturels sénégalais : résultats de 10 années d’observations de 1974 à 1984. Dakar, ISRA/LNERV, 1984. 50 p., tableaux et annexes.

30. VALENZA (J.), DIALLU (A.K.). Etude des päturages du Nord Sénegal. Maisons-Alfort, IEMVT, Dakar-Hann, LNERV, 1972. 311 p. 1 carte couleur à $1 / 200000$ en 3 feuilles. (Etude agrostologique $n^{\circ} 34$ )

AKPO (L.E.), GROUZIS (M.), GASTON (A.). Pluviosidad y productividad de los pastos del área de Wiidu Thiengoli en Ferlo (Norte de Senegal). Estimación de las cargas frecuenciales. Revue Élev. Méd. vét. Pays trop., 1993, 46 (4) : 675-681

A partir de observaciones realizadas durante 15 años (1974-1988) alrededor de la zona de forraje de Wiidu Thiendoli, situada en el norte de Senegal, se estudiaron las características pluviométricas y su influencia subre la producción de pastos. Los resultados muestran una fuerte variabilidad temporal tanto de las precipitaciones como de la producción. Un modelo simple de simulación de esta producción, fue elaborado en función de la pluviosidad. Si se comparan a las cargas actuales, las cargas frecuenciales establecidas a partir de esta formulacion y de la serie cronológica de las lluvias obtenidas en Wiidu Thiengoli, se observa un déficit en la disponibilidad forrajera, más importante durante la estación seca. Este tipo de situación representa una amenaza para los grupos tradicionales de ganaderos que explotan este forraje, ya que la vegetación natural constituye la casi totalidad de la alimentación de los hatos en estos sistemas de producción.

Palabras claves: Tierras de pastos - Densidad de pastores - Lluvia - Pastoreo-Transhumancia - Sahel - Senegal. 